Baxter's suggestion-namely, to change the warnings on packets of cotton tipped swabs and warn the mothers of possible dangers.

As it is not practical and also not generally advisable to use recurrent syringing for the removal of wax plugs in children, the use of olive oil or other commercially available wax softener ear drops (Waxsol, Cerumol) should be encouraged. The softened wax plugs can then be easily cleaned with either cotton swabs or ordinary cleaning tissue paper.

D KUMAR

Child Health Services, Sheffield Health Authority, Sheffield

\section{Risks of intracranial haematoma in head injured adults}

SIR,-Because the elderly are liable to subdural haematoma even with trivial head injury ${ }^{1}$ it would be useful to know what proportion of patients aged over 60 in the study by $\mathrm{Mr}$ A D Mendelow and others (22 October, p 1173) were found to have intracranial haemorrhage without skull fracture.

O M P JOLOBE

\section{Geriatric Department,}

Birmingham B18 $7 \mathrm{QH}^{2}$ ' Brain R. Subdural haematoma. In: Walton JN, reviser.
Brain's diseases of the nervous system. 8th ed.
Oxford: Oxford Medical Publications, 1977.

${ }^{*}{ }^{*}$ We sent a copy of this letter to the authors, who reply below.-ED, $B M \mathcal{F}$.

SIR,-Although it is true that the elderly may develop a chronic subdural haematoma after a trival injury, our study dealt with a very different problem-the acute traumatic intracranial haematoma.

Of 409 patients known to be under 60 years of age $100(24 \%)$ did not have a fracture, and of 133 over the age of 60 years $32\left(24^{\circ}{ }_{0}\right)$ did not have a fracture. The risk factors that we reported may therefore be applied to adults of all ages.

A D MENDELOW Graham TEASDALE GORDON MURRAY

Institute of Neurological

Sciences,

Southern General Hospital,

Glasgow G51 4TF

\section{Oral iron and the bioavailability of zinc}

SIR,-Though we would not dispute the finding of $\mathrm{Dr} \mathrm{N} \mathrm{J}$ Meadows and others (8 October, p 1013) that inorganic iron in the form of Pregaday tablets (ferrous fumarate and folic acid) reduces the absorption of pharmacological amounts of inorganic zinc, the relevance of this to the absorption of dietary zinc is uncertain.

We would like to draw attention to four points perhaps worthy of consideration. Firstly, using a series of "typical" meals and an intubation technique for intraluminal sampling, Matseshe and his colleagues showed that the sites of absorption of iron and zinc were not identical, iron being absorbed preferentially from the duodenum and zinc at a more distal site. ${ }^{1}$

Secondly, the study by Solomons and Jacobs, quoted by Dr Meadows and his col- leagues, does show impaired bioavailability of inorganic zinc in the presence of inorganic iron, but also shows that the absorption of $54 \mathrm{mg}$ of "organic zinc" in the form of Atlantic oysters is unaffected by $100 \mathrm{mg}$ of inorganic iron (iron to zinc molar ratio $2: 1$ ). ${ }^{2}$ It should be noted that this study and that of Dr Meadows and others use single test doses of zinc that are more than three times the recommended daily allowance ${ }^{3}$ and are therefore non-physiological. Thirdly, from the recommended daily allowance for zinc, the expected intake for each meal should be about $5 \mathrm{mg}$. Payton et al, using a dose of $6 \mathrm{mg}$ zinc chloride, labelled with the radionuclide zinc 65 , showed that an iron to zinc molar ratio of 5 to 1 did not appreciably lower zinc absorption. ${ }^{4}$

Finally, work by Tamura $e t$ al has suggested a relation between zinc and folate metabolism, ${ }^{5}$ and therefore the possibility that the folate component of Pregaday may influence zinc absorption cannot be ignored.

We agree with Dr Meadows and his colleagues that further research is necessary before zinc supplementation in pregnancy can be recommended. Even if it is established that iron does compete with zinc, the effect of any zinc supplementation on copper metabolism would then need to be considered. ${ }^{6}$

\section{Department of Surgery,}

Gartnavel General Hospital, Glasgow G12 0YN

K MitCheli

Department of Clinical Physics

and Bioengineering.

West of Scotland Health Boards,

Glasgow

Matseshe JW, Phillips SF, Malagelada J-R, McCall JT. Recovery of dietary iron and zinc from the ent meals and supplements. Am $\mathcal{f}$ Clin Nutr 1980 33:1946-53.

Solomons NW, Jacob RA. Studies on the bioavailability of zinc in humans: effects of heme and nonheme on the absorption of zinc. Am f Clin Nutr $1981 ; 34: 475-82$

Food and Nutrition Board. Recommended dietary Sciences, 1980 .

Payton KB, Chamberlain MJ, Valberg LS. Technique for th determination of human zinc absorption from measurement of radioactivity in a fecal sample or the gy $1982 ; 83: 1264-70$

5 Tamura T, Shane B, Baer MT, King JC, Margen S, Stokstad ELR. Absorption of mono- and polyglutamyl folates in zinc-depleted man. Am $\mathcal{F}$ Clin Prasad AS, Brewer Gs, cupremia induced by zinc therapy. ${ }_{A M}$. Hypo240:2166.

\section{Pulmonary hypertension in} systemic lupus erythematosus

SIR,-The coexistence of hypertension and systemic lupus erythematosus is rare. ${ }^{1} \mathrm{We}$ have recently reported a case similar to those of Dr R A Asherson and others (8 October, p 1024). ${ }^{2}$

A 26 year old woman had had typical systemic lupus erythematosus for four years. She had also had multiple spontaneous abortions and Raynaud's phenomenon. Later she developed cough, haemoptysis, and progressive dyspnoea and died after six months despite treatment. She showed the overt clinical and radiological findings of cor pulmonale. Necropsy showed multiple thrombi of varying ages in the small and large branches of the pulmonary artery (micro- and macrothrombi). With the exception of right ventricular hypertrophy no cardiac disease, diffuse interstitial pulmonary fibrosis, or pulmonary vasculitis could be detected. Histological examination of the kidneys and spleen showed specific findings of systemic lupus erythematosus. Presence of lupus anticoagulant was not investigated but a Venereal Disease Research Laboratory test was strongly positive.

As seen in this case severe pulmonary hypertension may develop in systemic lupus erythematosus and may be fatal. Dr Asherson and his colleagues have suggested pulmonary microthrombi as a possible mechanism for the development of pulmonary hypertension. Our experience with this patient supports their opinion. Although pulmonary hypertension may be related to diffuse interstitial pulmonary fibrosis, pulmonary vasculitis, and vascular changes of the primary pulmonary hypertension type, we think that pulmonary thromboembolism has a prominent role in most cases. The fact that venous syndromes and pulmonary thromboembolism in systemic lupus erythematosus are not as rare as once thought supports this idea. ${ }^{3}$ A hypercoagulable state due to lupus anticoagulant has been suggested as an important factor in the development of pulmonary hypertension resulting from pulmonary thromboembolism in systemic lupus erythematosus ( 15 October, p 1088). Additionally, recent reports have suggested a possible relation between lupus anticoagulant and arachidonic acid metabolism, and the inhibitory effect of the above mentioned antibodies on the production of prostacyclin has been discussed. ${ }^{4}$

Ahmet Oktay Ali OTO

Division of Cardiology,

Hacettepe University School of

Medicine,

Ankara, Turkey

Perez HD, Kramer N. Pulmonary hypertension in SLE: report of four cases and review of the literature. Semin Arthritis Rheum 1981;11:177-81. Oktay A, Oto A, Ruacan S, Telatar H. Pulmonary hypertension and systemic lupus erythematosus A case report. Hacettepe Medical fournal 1980 13:103-11.

odman DD, Urowitz MB. Venous syndromes and pulmonary emb

4 McVery BA, Machin SJ, Parry H, Goldstone AH. Reduced prostacyclin activity in SLE. Ann Rheum Dis $1980 ; 39: 524-5$.

\section{Infant chlamydial pneumonia}

SIR,-We hope that the report by Dr Anne A Attenburrow (22 October, p 1219) of fatal chlamydial disease in an infant may further motivate antenatal diagnosis of chlamydial infection. Though it is not yet feasible to provide full antenatal screening for Chlamydia trachomatis, medical and sociological data may be used to identify women at risk.

In the first 12 months of investigating severe neonatal eye infection in this hospital, with correlation of sociological risk factors, ${ }^{1}$ 13 cases of chlamydial ophthalmia neonatorum were diagnosed in 84 infants screened. These included six samples from general practice, of which two were positive. The peak onset of signs of neonatal inclusion conjunctivitis was on the eighth day of life (range 3rd to 13th day): only one child developed signs after the 10th day. None of these babies were proved to have a chlamydial pneumonitis.

A review of the cases prompted several observations. Firstly, late attendance at the antenatal clinic may be an indication for chlamydial screening: all but two of the mothers of the 13 affected infants failed to receive antenatal care until the 20th week of pregnancy; five did not receive care until the 